\title{
Novel Route for Synthesis of Anti - Hyperglycaemic Activity of Thiazolidine 2,4- Dione Derivatives As A Mannich Bases
}

Ramakrishna Vellalacheruvu*1, R Sai Leela ${ }^{2}$ and L K Ravindranath ${ }^{1}$

${ }^{1}$ Department of Chemistry, SK University, India

${ }^{2}$ College of Pharmacy, SK University, India

Submission: July 28, 2017; Published: August 21, 2017

"Corresponding author: Ramakrishna Vellalacheruvu; Department of Chemistry, Sri Krishna Devaraya University, Anantapur, Andhra Pradesh, India, Tel: 9493268448; Email: vellalacheruv@gmail.com

\section{Abstract}

The mannish bases of Thiozolidine 2,4 -dione derivatives has come to lime light due to their multi functional biological activities. Thiazolidine2,4-dione is an extensively explored hetero cyclic nucleus for designing of novel agents implicated for a wide variety of pathophysiological conditions, that is, diabetes, diabetic complications ,cancer, arthritis, inflammation, microbial infection, and melanoma. Present work, synthesise quinoline attached imidozoline derivative using $(3+2)$ cycloaddition via imine of quinoline and TOSMIC. These derivatives were converted to mannich bases of thiozolidine 2,4 one using knoevenagel condensation. The sulfonyl derivatives of thiozolidine 2, 4 -dione were also synthesized and characterized by using alkylation conditions.

Keywords: Imidazole derivatives; Thiozolidine 2,4 one nucleus; TOSMIC; Combi- flash Chromatography

\section{Introduction}

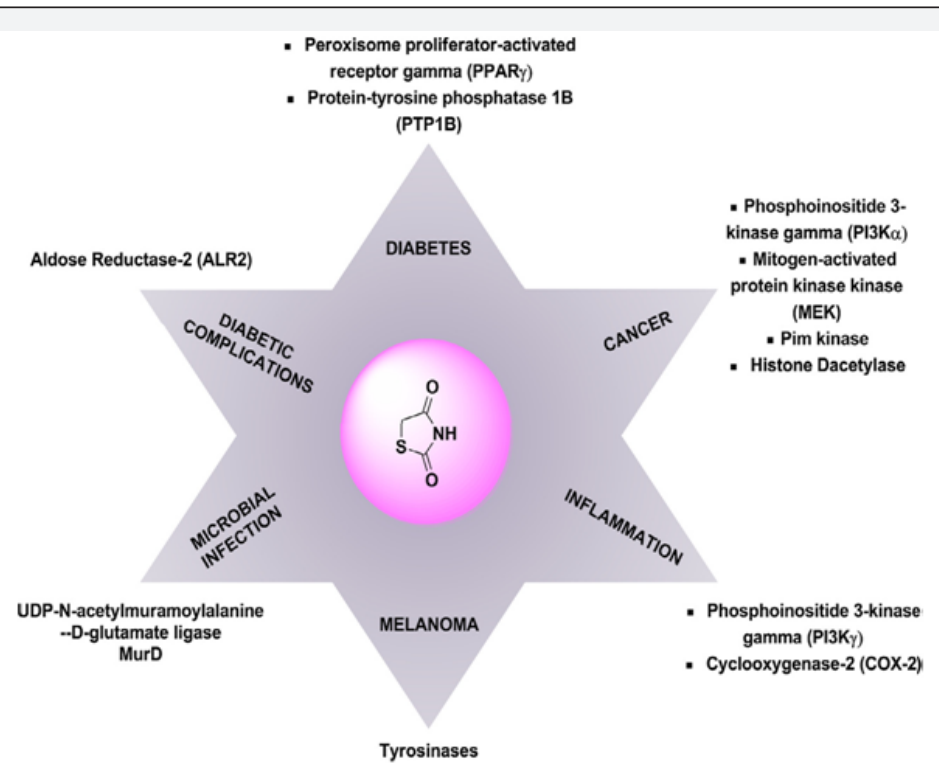

Figure 1 


\section{Organic and Medicinal Chemistry International Journal}

Thiazolidine 2,4 dione (TZD) is a vital nucleolus in heterocyclic chemistry. TZD shows multidirectional phamocodynamical activities such as Anti-hyperglycemic (glitazone drugs), anti-cancer, anti microbial, anti-arthritic. Due to multidirectional pathological actions, huge explored research work has been attempted and still efforts under progress for drug candidates. The metabolic disorder of diabetic is now a day's shows major impact on human beings throughout world. The Thiazolidine 2,4-dione (TZD) derivatives act as a drug candidates such as rosiglitagone, pioglitazone, lobigli tazone, enaglitazone, netoglitazone, ozoline, daragletazone, troglitazone etc. TZD derivatives not only confine for treatment of metabolic disorder diabetic, it o shows as an inflammatory agents, and anti- cancer and for treatment of melanoma. Due to importance of TZD, derivatives many scientists have been developed various routes for synthesis. Om silakari et al. developed different TZD derivatives and evolutes their biological activity (Figure 1). Ivanildo Mangueira, da Silva and co workers [1] developed TZD derivatives using Knoevenagel condensation (Figure 2). Boja Poojary and co workers [2] synthesized and characterization of Antimicrobial activity of novel derivatives (Figure 3). Archana Kapoor and Neha Khare [3] synthesized various mannich bases of Antibacterial and antifungal activity of 2,4-thiazolidinedione and rhodanine (Figure 4). Many routes has been developed for synthesis of 2,4thiozolidinone. The Thiazolidine 2,4 dione having many active sites. Thiazolidine 2,4 ,dione nucleus numbering is given as fallows (Figure 5).

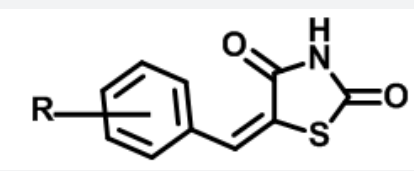

Figure 2: TZD derivatives using Knoevenagel condensation.

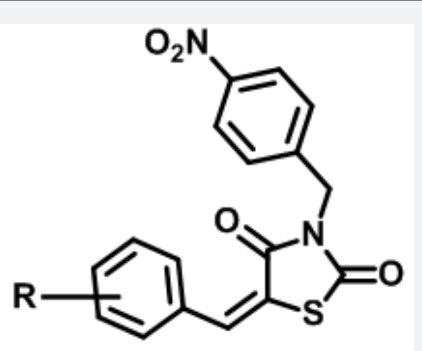

Figure 3: Synthesized and characterization of Antimicrobial activity of novel derivatives.

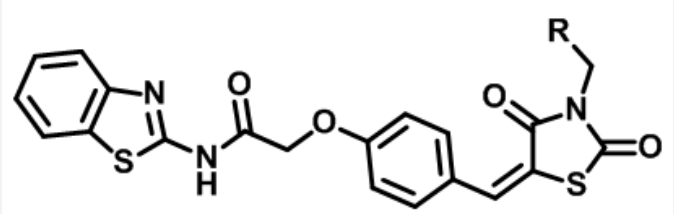

Figure 4: synthesized various mannich bases of Antibacterial and antifungal activity of 2,4-thiazolidinedione and rhodanine.

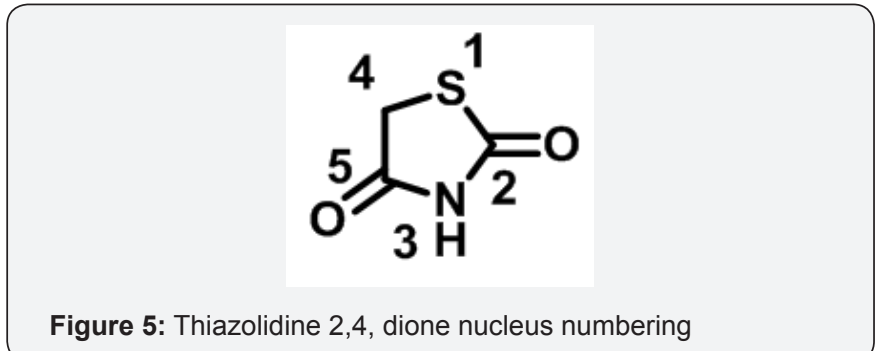

Materials and Methods

All reagents and starting material were procured from commercial sources (Aldrich, Alfa Aesar). Solvents were thoroughly dried before use. THF and toluene were dried using sodium metal and benzophenone.DMF was dried using CaH. The new compounds were fully characterized using Analytical methods like IR, NMR (Bruker). The melting points were recorded using on a (WRS-1A) Digital Melting Point Apparatus without correction. Infrared spectra were taken using an AVATAR 370 FT-IR spectrometer. HNMR, CNMR spectra were recorded with a Bruker spectrometer operating at $400 \mathrm{MHz}$ used as a Trimethyl silane reference and values recorded in ppm. The progress of reaction was monitored using TLC system and $I_{2}$ spray and $\mathrm{KMnO}_{4}$ TLC strain. The crude compounds were purified using column chromatography (100-200 mesh silica) and Combi-flash chromatography. The hydrogenolysis process was carried out using parr shaker [4-19].

\section{Objective of this Research}

Present work is related to develop new synthetic route for preparation of the quinoline containing thiozolidin-4one attached 1,3, 4 oxa diazole nucleus and thiazolidin-4-one attached benz imidazole and benz thiozole and benzoxazole derivatives and thoroughly characterized. The scaffolds of 2-(8-((5-(4- substituted phenyl)-1,3,4-oxadiazol-2-yl)methoxy) quinolin-5-yl)-3-(4-(trifluoromethyl)phenyl)thiazolidin-4one(7a-h)weresynthesized and characterized.

\section{Experimental Methods}

In this research work, we prepared below compounds and mentioned in step wise manner.

a) Step-1: (Z)-N-((8-(benzyloxy)quinolin-5-yl)methylene)-4(trifluoromethyl)aniline (2)

b) Step-2: 8-(benzyloxy)-5-(1-(4-(trifluoromethyl) phenyl)1H-imidazol-5-yl) quinoline (3).

c) Step-3: 5-(1-(4-(trifluoromethyl) phenyl)-1H-imidazol-5yl) quinolin-8-ol (4).

d) Step-4: 2-((5-(1-(4-(trifluoromethyl) phenyl)-1H-imidazol5-yl) quinolin-8-yl) oxy) acetaldehyde (5).

e) Step-5: 5-(5-(1-(4-(trifluoromethyl)phenyl)-1H-imidazol5-yl)quinolin-8-oxy)methylene)thiazolidine-2,4-dione (6). 


\section{Organic and Medicinal Chemistry International Journal}

f) Step-6: 3-(Amine substituted methyl)-5-(2-((5-(1-(4(trifluoromethyl) phenyl)-1H-imidazol-5-yl)quinolin-8-yl)oxy) ethylidene)thiazolidine-2,4-dione (7a-f). g) Step-7: 3-(sulfonyl-derivatives)-5-(2-((5-(1-(4-(trifluoromethyl) phenyl)-1H-imidazol-5-yl) quinolin-8-yl)oxy)ethylidene)thiazolidine2,4-dione (8a-f) (Figure 6).

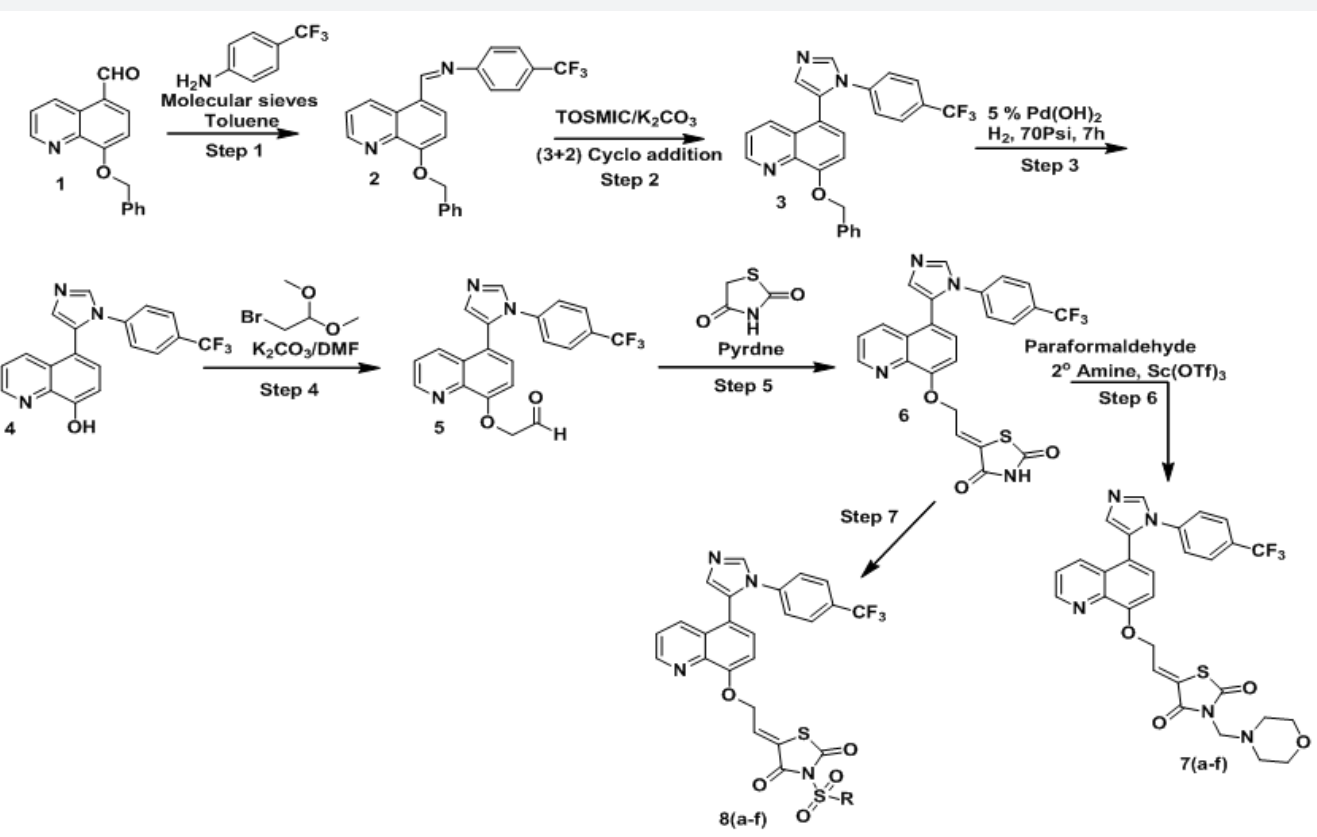

Reaction Conditions: Step 1) Molecular sieves, Toluene, $100{ }^{\circ} \mathrm{C}, 10 \mathrm{~h}$, Step 2) TOSMIC $/ \mathrm{K}_{2} \mathrm{CO}_{3}, 0{ }^{\circ} \mathrm{C}, 16 \mathrm{~h}$, Step 3) $5 \% \mathrm{Pd}(\mathrm{OH})_{2}, \mathrm{H}_{2}$ 70Psi, $3 \mathrm{~h}$, Step 4) 2-bromo-1,1-dimethoxy ethane, $\mathrm{K}_{2} \mathrm{CO}_{3} / \mathrm{DMF}$, 5h, Step 5) thiazolidine-2,4-dione, Piperdine, 90 $\left.{ }^{\circ} \mathrm{C}, 6 \mathrm{~h}, \mathrm{Step} 6\right) \mathrm{Para}^{\circ}$ formaldehyde $/ 20$ Amine, $\mathrm{Sc}(\mathrm{OTf})_{3} \mathrm{EtOH}, 90^{\circ} \mathrm{C}, 8 \mathrm{~h}$.

Figure 6: Scheme:

\section{Reaction mechanism for Step 2 (Figure 7)}

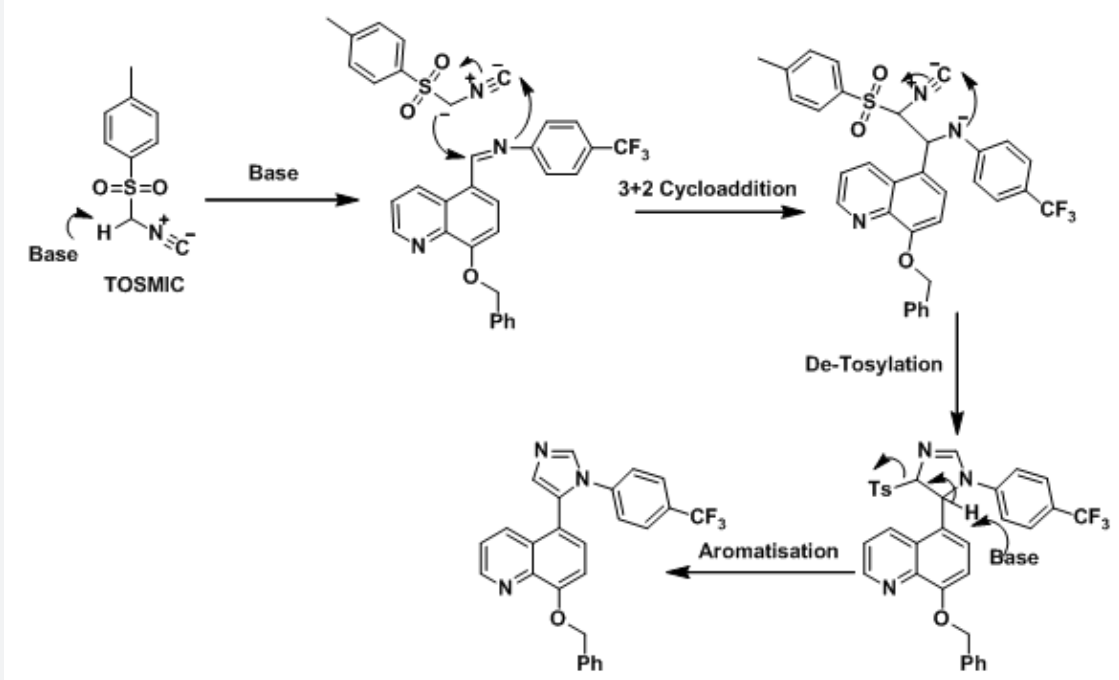

Figure 7: Reaction mechanism for Step 2

\section{Step 1: (Z)-N-((8-(benzyloxy) quinolin-5-yl) methylene)-4-(trifluoromethyl) aniline(2)}

8-(benzyloxy) quinoline-5-carbaldehyde (10 g, $0.038 \mathrm{~mol}$ ), 4-(trifluoromethyl) aniline $(6.5 \mathrm{~g}, 0.039 \mathrm{~mol})$ in dry toluene (
$100 \mathrm{~mL}$ ) was added freshly dried molecular sieves and refluxed for $10 \mathrm{~h}$ under $\mathrm{N}_{2}$ atm. The progress of reaction was monitored by TLC. After completion of starting material, toluene was evaporated under vacuum to gave crude residue of Compound- 2 


\section{Organic and Medicinal Chemistry International Journal}

(15 g) as a solid ( white colour). The crude was carried to next step (Figure 8).

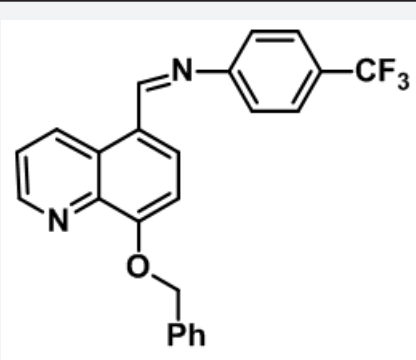

Figure 8: (Z)-N-((8-(benzyloxy) quinolin-5-yl)methylene)-4(trifluoromethyl) aniline(2)

Step 2 : 8-(benzyloxy)-5-(1-(4-(trifluoromethyl) phenyl)-1H-imidazol-5-yl)quinoline(3)

(Z)-N-((8-(benzyloxy)

quinolin-5-yl)methylene)-4(trifluoromethyl) aniline(2) (15 g, $0.036 \mathrm{~mol}$ )was dissolved in Dry DMF ( $80 \mathrm{~mL})$ and cooled to $\mathrm{O}^{\circ} \mathrm{C}$. To that dried $\mathrm{K}_{2} \mathrm{CO}_{3}(15 \mathrm{~g}$. $108 \mathrm{~mol}$ ) and Toluene methyl isocyanide (7.02 g, $0.036 \mathrm{~mol}$ ) was added and warm to room temperature and stirred for $16 \mathrm{~h}$. The progress of reaction was monitored by TLC. After completion, reaction mixture was poured in ice cold water $(100 \mathrm{~mL})$ and extracted with EtOAc ( $3 \times 100 \mathrm{~mL}$ ). The organic layer was separated and washed with brine solution, dried over anhydrous $\mathrm{Na}_{2} \mathrm{SO}_{4}$, filtered and evaporated under vacuum to give crude residue. The obtained crude product was purified by column chromatography (100-200 mesh silica, Eluent: 80\% EtOAc-Pet Ether) isolated 8-(benzyloxy)-5-(1-(4-(trifluoromethyl) phenyl)1H-imidazol-5-yl)quinoline(3) (10 g, yield: 64\%) as a solid (Pale yellow colour). M.p. 252-255 ${ }^{\circ} \mathrm{C}$. IR (KBr, cm-1): 3030, 1440, 1520, 1005, 691, 655. HNMR (d -DMSO, 400 mHz): 5.2 (s, 1H, $\left.-\mathrm{CH}_{2}\right), 7.1(\mathrm{~m}, 3 \mathrm{H}), 7.3-7.5(\mathrm{~m}, 7 \mathrm{H}), 7.6-7.7(\mathrm{~m}, 4 \mathrm{H}), 7.85(\mathrm{~d}, 1 \mathrm{H})$, $8.36(\mathrm{~d}, 1 \mathrm{H}), 8.85(\mathrm{~d}, 1 \mathrm{H})$ (Figure 9).

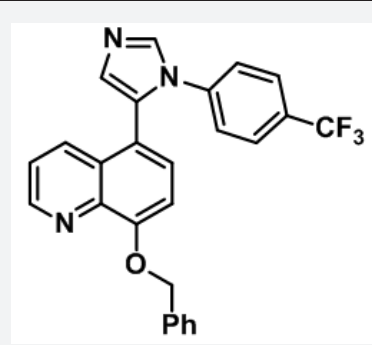

Figure 9: 8-(benzyloxy)-5-(1-(4-(trifluoromethyl)phenyl)-1Himidazol-5-yl)quinoline(3)

\section{Step 3 : 5-(1-(4-(trifluoromethyl)phenyl)-1H-imidazol- 5-yl)quinolin-8-ol (4)}

8-(benzyloxy)-5-(1-(4-(trifluoromethyl)phenyl)-1Himidazol-5-yl)quinoline(3) (10 g, $0.022 \mathrm{~mol}$ ) in $\mathrm{MeOH}$ (100 $\mathrm{mL}$ ) was added $5 \%$ Palladium hydroxide on carbon (1 g, cat) and hydrozinated at 70 Psi under parr shaker for $3 \mathrm{~h}$ at room temperature. The progress of reaction was monitored by TLC. After completion, reaction mixture was filtered on cellite bed and thoroughly washed with $\mathrm{MeOH}(2 \times 75 \mathrm{~mL})$. The $\mathrm{MeOH}$ layer were collected and evaperated under vaccum to gave 5-(1-(4-(trifluoromethyl)phenyl)-1H-imidazol-5-yl)quinolin-8ol (4)(7g, yield : 86\%) as a solid (white colour)). M.p. 280-285 ${ }^{\circ} \mathrm{C}$. IR (KBr, cm-1): 3620, 3014, , 1525, 1050, 691, 620 . ${ }^{1}$ HNMR (

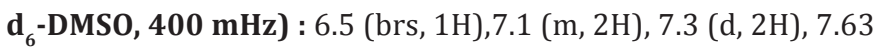
$(\mathrm{m}, 4 \mathrm{H}), 7.8(\mathrm{~d}, 1 \mathrm{H}), 8.35(\mathrm{~d}, 1 \mathrm{H}), 8.8(\mathrm{~d}, 1 \mathrm{H})$ (Figure 10).

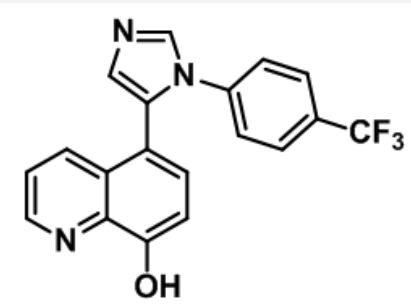

Figure 10: 5-(1-(4-(trifluoromethyl)phenyl)-1H-imidazol-5-yl) quinolin-8-ol (4)

Step 4 : 2-((5-(1-(4-(trifluoromethyl)phenyl)-1Himidazol-5-yl)quinolin-8-1)oxy)acetaldehyde (5)

5-(1-(4-(trifluoromethyl) phenyl)-1H-imidazol-5-yl) quinolin-8-ol (4) (7 g, $0.017 \mathrm{~mol}$ ) in Dry DMF ( $70 \mathrm{~mL}$ ) was added $\mathrm{K}_{2} \mathrm{CO}_{3}(9.7 \mathrm{~g}, 0.07 \mathrm{~mol}, 4 \mathrm{eq})$ and stirred at $\mathrm{rt}$ for $30 \mathrm{~min}$. To that a solution of 2-bromo-1,1-dimethoxy ethane( 1.2 eq) in DMF (20 $\mathrm{mL}$ ) was added drop wise at $0^{\circ} \mathrm{C}$ and stirred for $5 \mathrm{~h}$. The progress of reaction was monitored by TLC. After completion, reaction mixture was filtered on cellite bed and washed with DMF (10 $\mathrm{mL}$ ). The Reaction mixture was poured in ice cold water (200 $\mathrm{mL}$ ) and stirred for $20 \mathrm{~min}$. The reaction mixture was acidified aq $\mathrm{NaHSO}_{3}$ solution up to $\mathrm{P}^{\mathrm{H}}-5$ and extracted with EtOAc $(2 \times 200$ $\mathrm{mL}$ ). The aqueous layer was collected and basified up to $\mathrm{P}^{\mathrm{H}}-8$ with sat aq $\mathrm{NaHCO}_{3}$ sol. The aqueous layer was extracted with EtOAc ( 3 x $100 \mathrm{~mL}$ ). The organic layer were collected and dried over anhydrous $\mathrm{Na}_{2} \mathrm{SO}_{4}$, filtered and evaporated under vacuum to gave 2-((5-(1-(4-(trifluoromethyl)phenyl)-1H-imidazol-5yl)quinolin-8-yl)oxy) acetaldehyde (5) (5g) as a solid( white colour). M.p. $200-205{ }^{\circ} \mathrm{C}$. IR (KBr, cm-1): 3602, 3014, 1712, 1646, 1503, 1050, 691, 644. ${ }^{1}$ HNMR (d -DMSO, 400 mHz) : 5.2 $(\mathrm{s}, 2 \mathrm{H}), 7.1(\mathrm{~m}, 2 \mathrm{H}), 7.3(\mathrm{~d}, 2 \mathrm{H}), 7.9(\mathrm{~d}, 1 \mathrm{H}), 8.36(\mathrm{~d}, 1 \mathrm{H}), 8.82(\mathrm{~d}$, 1H), 9.6(s, 1H) (Figure 11).

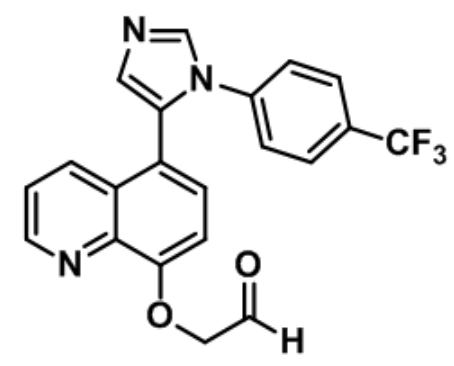

Figure 11: 2-((5-(1-(4-(trifluoromethyl)phenyl)-1H-imidazol-5yl)quinolin-8-yl)oxy) acetal dehyde (5) 
Step 5: 5-(2-((5-(1-(4-(trifluoromethyl)phenyl)1H-imidazol-5-yl)quinolin-8-yl)oxy) ethylidene) thiazolidine-2,4-dione (5)

To a mixture of 2-((5-(1-(4-(trifluoromethyl)phenyl)-1Himidazol-5-yl)quinolin-8-yl)oxy) acetaldehyde (5) (5g, 0.012 mol), thiazolidine-2,4-dione (1.62 g, $0.013 \mathrm{~mol})$ in EtOH (50 mL) was added piperdine ( $2 \mathrm{~mL}$ ) and heated at $90{ }^{\circ} \mathrm{C}$ for $6 \mathrm{~h}$. The progress of reaction was monitored by TLC. After completion, Reaction mixture was evaporated under vacuum to gave crude residue. The residue was diisolve in water $(100 \mathrm{~mL})$ and filtered under vaccum and dried to gave (Z)-5-(2-((5-)(1-(4(trifluoromethyl) phenyl)-1H-imidazol-5-yl)quinolin-8-yl)oxy) ethylidene) thiazolidine-2,4-dione (6) ( $5.5 \mathrm{~g}$, Yield: $88 \%$ ) as a solid ( brown colour).M.p:240-243 ${ }^{\circ} \mathrm{C}$. IR (KBr, cm-1): 3050, 1725, 1650, 1503, 1050, 691, 644. ${ }^{1}$ HNMR ( d 6 -DMSO, $400 \mathrm{mHz}$ ) : $4.6(\mathrm{dd}, 1 \mathrm{H}), 4.61(\mathrm{dd}, 1 \mathrm{H}), 6.15(\mathrm{dd}, 1 \mathrm{H}), 7.12(\mathrm{~m}, 2 \mathrm{H}), 7.3(\mathrm{~d}$, $2 \mathrm{H}), 7.6-7.65(\mathrm{~m}, 4 \mathrm{H}), 7.9(\mathrm{~d}, 1 \mathrm{H}), 8.4(\mathrm{~d}, 1 \mathrm{H}), 8.6$ (brs, $1 \mathrm{H}), 8.87$ (d, 1H) (Figure 12).

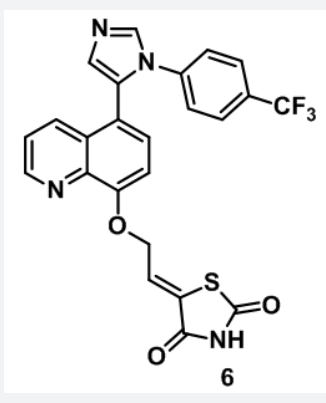

Figure 12: 5-(2-((5-(1-(4-(trifluoromethyl)phenyl)-1H-imidazol5-yl)quinolin-8-yl)oxy) ethylidene) thiazolidine-2,4-dione (5)

Step 6 : 3-( Amino substituted methyl)-5-(2-((5-(1-) (4(trifluoromethyl)phenyl)-1H-imidazol-5-yl)quinolin8-yl)oxy)ethylidene)thiazolidine-2,4-dione \&7(a-f)

To a mixture of (Z)-5-(2-((5-(1-(4-(trifluoromethyl) phenyl)1H-imidazol-5-yl)quinolin-8-yl)oxy)ethylidene) thiazolidine2,4-dione (6) ( $500 \mathrm{mg}$, ), 2o Amine (1.1 eq), Para formal dehyde $(3 \mathrm{eq})$ in EtOH $(50 \mathrm{~mL})$ was added $\mathrm{Sc}(\mathrm{OTf})_{3}(0.1 \mathrm{eq})$ and heated for $8 \mathrm{~h}$. The progress of reaction was monitored by TLC. After completion, EtOH was evaporated under vaccum to give crude product. The crude was purified by reverse-phase column chromatography (C18 silica, Eluent: 30\% ACN-MeOH- $\mathrm{H}_{2} \mathrm{O}$, $0.01 \%$ TFA) isolated (Z)-3-(Amino substituted methyl)-5-(2-((5(1-(4-(trifluoromethyl)phenyl)-1H-imidazol-5-yl)quinolin-8-yl) oxy) ethylidene) thiazolidine-2,4-dione \&7(a-f). 1H NMR spectra of 7 (a-f) was given below Table 1 \& Figure 13.

Table 1: $1 \mathrm{H}$ NMR spectra.

\begin{tabular}{|l|l|l|l|l|l|l|}
\hline Comp & $\mathbf{7 a}$ & $\mathbf{7 b}$ & $\mathbf{7 c}$ & $\mathbf{7 d}$ & $\mathbf{7 e}$ & $\mathbf{7 f}$ \\
\hline $\mathbf{X}$ & C=0 & N-Me & N-Boc & N-Et & 0 & S \\
\hline
\end{tabular}

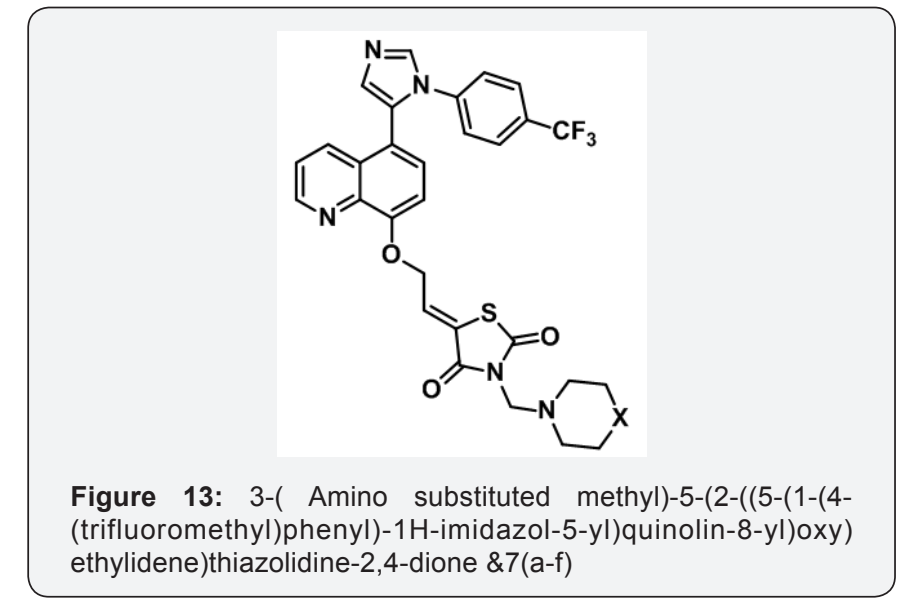

a) 3-((4-oxopiperidin-1-yl)methyl)-5-(2-((5-)(1-(4(trifluoromethyl)phenyl)-1H-imidazol-5-yl)quinolin-8-yl) oxy)ethylidene)thiazolidine-2,4-dione (7a): M.p. 280-283 ${ }^{\circ} \mathrm{C}$. IR (KBr, cm-1): 3050, 3010, 1720, 1655, 1600, 1320,770,

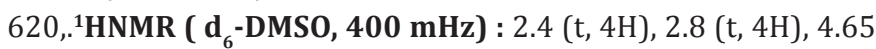
(s, 2H, ) 4. 68(dd,2H), $4.7(\mathrm{dd}, 1 \mathrm{H}), 5.1(\mathrm{~d}, 2 \mathrm{H}), 6.8(\mathrm{dd}, 1 \mathrm{H}), 7.1$ (m, 1H), $7.3(\mathrm{~d}, 2 \mathrm{H}), 7.6(\mathrm{~m}, 4 \mathrm{H}), 8.0(\mathrm{~d}, 1 \mathrm{H}), 8.43(\mathrm{~d}, 1 \mathrm{H}), 8.81(\mathrm{~d}$, 1H). ${ }^{13}$ CNMR ( $d_{6}$-DMSO, 400 mHz) : 45, 53, 65, 108, 120, 122, 124, 124.5, 131, 135, 139, 145,150,164, 173, 190.

b) 3-((4-methylpiperazin-1-yl)methyl)-5-(2-((5-(1-(4(trifluoromethyl)phenyl)-1H-imidazol-5-yl)quinolin-8-yl) oxy)ethylidene)thiazolidine-2,4-dione (7b): M.p. $290-292^{\circ} \mathrm{C}$. IR (KBr, cm-1): 3350, 3050, 1660,1610, 1320,750, 625, ${ }^{1}$ HNMR (

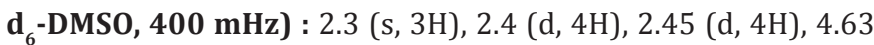
(s, 2H,), $4.66(\mathrm{dd}, 1 \mathrm{H}), 4.67(\mathrm{dd}, 1 \mathrm{H}), 6.81(\mathrm{dd}, 1 \mathrm{H}), 7.1(\mathrm{~m}, 2 \mathrm{H})$, $7.32(\mathrm{~d}, 2 \mathrm{H}), 7.6(\mathrm{~m}, 4 \mathrm{H}), 8.03(\mathrm{~d}, 1 \mathrm{H}), 8.44(\mathrm{~d}, 1 \mathrm{H}), 8.81(\mathrm{~d}, 1 \mathrm{H})$. C-NMR ( d 6 -DMSO, 400 mHz) : 47, 53, 58, 65, 107, 121, 122, 124, 124.5, 127, 130, 134, 138, 145,150,163, 174.

c) tert-butyl 4 - ( ( 2,4 - d i oxo - 5 - ( 2 - ( ( 5 - ( 11 - ( 4 (trifluoromethyl)phenyl)-1H-imidazol-5-yl)quinolin-8-yl) oxy)ethylidene)thiazolidin-3-yl)methyl)piperazine-1carboxylate (7c): M.p. $260-2262^{\circ} \mathrm{C}$. IR (KBr, cm-1): , 3014, 1713, 1650, 1620, 1505, 1310, 1050, 698, 655, HNMR ( d $\mathbf{d}_{6}$-DMSO, 400 mHz) : $1.4(\mathrm{~s}, 9 \mathrm{H}), 2.5(\mathrm{t}, 4 \mathrm{H}), 3.1(\mathrm{t}, 4 \mathrm{H}), 2.45(\mathrm{~d}, 4 \mathrm{H}), 4.5(\mathrm{~s}, 2 \mathrm{H}$,$) ,$ $4.68(\mathrm{dd}, 1 \mathrm{H}), 4.69(\mathrm{dd}, 1 \mathrm{H}), 6.83(\mathrm{dd}, 1 \mathrm{H}), 7.1(\mathrm{~m}, 2 \mathrm{H}), 7.32(\mathrm{~d}$, 2H), $7.62(\mathrm{~m}, 4 \mathrm{H}), 7.9(\mathrm{~d}, 1 \mathrm{H}), 8.42(\mathrm{~d}, 1 \mathrm{H}), 8.82(\mathrm{~d}, 1 \mathrm{H}) .{ }^{13}$ C-NMR ( d -DMSO, $400 \mathrm{mHz}$ : : 31, 44, 52, 65, 78, 107, 121, 124, 127, 130, 132, 139, 145,154,162, 174.

d) 3-((4-ethylpiperazin-1-yl)methyl)-5-(2-((5-(1-(4(trifluoromethyl)phenyl)-1H-imidazol-5-yl)quinolin-8-yl) oxy)ethylidene)thiazolidine-2,4-dione (7d): M.p. 290-292 ${ }^{\circ} \mathrm{C}$. IR (KBr, cm-1): 3350, 3020, 1680,1620, 1330,750, 625, ${ }^{1}$ HNMR ( d6-DMSO, $400 \mathrm{mHz}$ ) : 1.2 (t, 3H ), $2.5(\mathrm{~m}, 10 \mathrm{H}), 4.5(\mathrm{~s}, 2 \mathrm{H}$ ), $4.67(\mathrm{~d}, 1 \mathrm{H}), 4.68(\mathrm{~d}, 1 \mathrm{H}), 6.80(\mathrm{dd}, 1 \mathrm{H}), 7.1(\mathrm{~m}, 2 \mathrm{H}), 7.3(\mathrm{~d}, 2 \mathrm{H})$, $7.62(\mathrm{~m}, 4 \mathrm{H}), 7.8(\mathrm{~d}, 1 \mathrm{H}), 8.42(\mathrm{~d}, 1 \mathrm{H}), 8.82(\mathrm{~d}, 1 \mathrm{H})$. C-NMR ( d $\mathbf{6}^{-}$ DMSO, $400 \mathrm{mHz}$ ) : 14, 50, 53,58,65, 108, 122, 124, 125, 130, 132, 145,149,165, 174 . 
e) 3 - ( morpholinomethyl) - 5 - ( 2 - ( ( 5 - ( 1 - ( 4 (trifluoromethyl)phenyl)-1H-imidazol-5-yl)quinolin-8-yl) oxy)ethylidene)thiazolidine-2,4-dione (7e): M.p. 280-282 ${ }^{\circ} \mathrm{C}$. IR (KBr, cm-1): 3016, 1720, 1650, 1503, 1300, 1050, 695, 650. ${ }^{1}$ HNMR ( $\mathbf{d}_{6}$-DMSO, $400 \mathrm{mHz}$ ) : $2.6(\mathrm{t}, 4 \mathrm{H}), 3.7(\mathrm{t}, 4 \mathrm{H}), 4.5$ $(\mathrm{s}, 2 \mathrm{H}),, 4.68(\mathrm{dd}, 1 \mathrm{H}), 4.69(\mathrm{dd}, 1 \mathrm{H}), 6.80(\mathrm{dd}, 1 \mathrm{H}), 7.15(\mathrm{~m}, 2 \mathrm{H})$, $7.25(\mathrm{~d}, 2 \mathrm{H}), 7.62(\mathrm{~m}, 4 \mathrm{H}), 7.9(\mathrm{~d}, 1 \mathrm{H}), 8.38(\mathrm{~d}, 1 \mathrm{H}), 8.89(\mathrm{~d}, 1 \mathrm{H})$. C-NMR (d-DMSO, 400 mHz) : 53,65, 66,107, 123, 124, 125, 130, 132, 139, 145,149,164, 178.

f) 3-(thiomorpholinomethyl) - 5 - ( 2 - ( ( 5 - ( 1 - ( 4 (trifluoromethyl)phenyl)-1H-imidazol-5-yl)quinolin-8-yl) oxy)ethylidene)thiazolidine-2,4-dione (7f): M.p. 272-275 ${ }^{\circ} \mathrm{C}$. IR (KBr, cm-1): 3080, 1730, 1645, 1520, 1310, 1125, 670, 660,

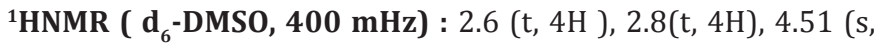
$2 \mathrm{H},), 4.66(\mathrm{dd}, 1 \mathrm{H}), 4.67(\mathrm{dd}, 1 \mathrm{H}), 6.81(\mathrm{dd}, 1 \mathrm{H}), 7.15(\mathrm{~m}, 2 \mathrm{H})$, $7.25(\mathrm{~d}, 2 \mathrm{H}), 7.62(\mathrm{~m}, 4 \mathrm{H}), 7.9(\mathrm{~d}, 1 \mathrm{H}), 8.37(\mathrm{~d}, 1 \mathrm{H}), 8.86(\mathrm{~d}, 1 \mathrm{H})$. C-NMR ( $\mathbf{d}_{6}$-DMSO, $400 \mathrm{mHz}$ ) : 27,58,63, 106, 122,123, 124, $125,130,131,135,139,145,149,164,174$.

Step 7: General procedure for -3-(sulfonyl derivative)5-(2-((5-(1-(4-trifluoromethyl)phenyl)-1H-imidazol5-yl)quinolin-8-yl)oxy)ethylidene)thiazolidine-2,4dione (8a-f)

5-(2-((5-(1-(4-(trifluoromethyl)phenyl)-1H-imidazol-5-yl) quinolin-8-yl)oxy)ethylidene) thiazolidine-2,4-dione (500 mg, $1.08 \mathrm{mmol}$ ) in Dry DMF(5 mL) was added $\mathrm{NaH}$ (3 eq) at $0{ }^{\circ} \mathrm{C}$ under $\mathrm{N}_{2} \mathrm{~atm}$ and stirred for $1 \mathrm{~h}$. To that sulfonyl chloride (1.1 eq) was added and stirred for $5 \mathrm{~h}$.The pogress of reaction was monitored by TLC. The reaction mixture was poured in aq sat $\mathrm{NaHCO}_{3}$ and stirred for $15 \mathrm{~min}$. The aq layer was extracted with $10 \% \mathrm{MeOH}-\mathrm{CHCl}_{3}\left(3 \times 25 \mathrm{ml}\right.$ ) and dried over anhydrous $\mathrm{Na}_{2} \mathrm{SO}_{4^{\prime}}$ filtered and evaporated under vaccum to gave crude product. The crude product was purified by Column chromatography (100-200 mesh silica) isolated 3-(sulfonyl derivative)-5-(2-((5(1-(4-(trifluoromethyl) phenyl)-1H-imidazol-5-yl)quinolin-8-yl) oxy) ethylidene) thiazolidine-2,4-dione (8a-f) (Figure 14 and Table 2).

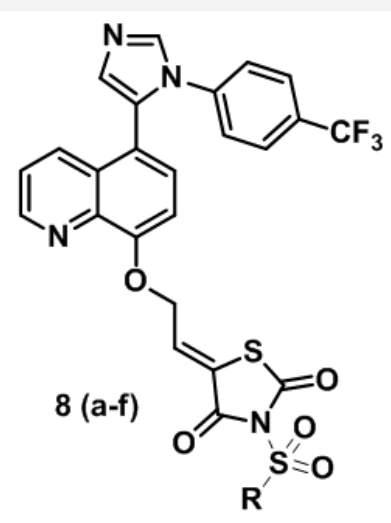

Figure 14: General procedure for -3-(sulfonyl derivative)-5-(2((5-(1-(4-(trifluoromethyl)phenyl)-1 H-imidazol-5-yl)quinolin-8$\mathrm{yl})$ oxy)ethylidene)thiazolidine-2,4-dione (8a-f).
Table 2:

\begin{tabular}{|c|c|c|c|c|c|c|}
\hline Comp & $8 a$ & $8 b$ & $8 c$ & 8d & $8 e$ & $8 f$ \\
\hline $\mathrm{R}$ & $\mathrm{Me}$ & Et & P-Tolyl & $\begin{array}{l}4-\mathrm{Cl}- \\
\mathrm{C}_{6} \mathrm{H}_{4}\end{array}$ & $4-\mathrm{Br}^{-}$ & $\begin{array}{l}4-\mathrm{NO}_{2}^{-} \\
\mathrm{C}_{6} \mathrm{H}_{4}\end{array}$ \\
\hline
\end{tabular}

i. $\quad 3$ - ( m e thylsulfonyl) - 5 - ( 2 - ( ( 5 - ( 1 - ( 4 (trifluoromethyl)phenyl)-1H-imidazol-5-yl)quinolin-8-yl) oxy)ethylidene)thiazolidine-2,4-dione(8a): M.p.280-283 ${ }^{\circ} \mathrm{C}$. IR (KBr, cm-1): 3040, 3025, 1730, 1645,1600, 1320,1070,770,715 620,. ${ }^{1}$ HNMR ( d d $^{6}$-DMSO, 400 mHz) : 2.8 (s, 3H), 4.67 (dd, 1H), $4.68(\mathrm{dd}, 1 \mathrm{H}), 6.8(\mathrm{dd}, 1 \mathrm{H}), 7.1(\mathrm{~m}, 2 \mathrm{H}), 7.3(\mathrm{~d}, 2 \mathrm{H}), 7.6(\mathrm{~m}, 4 \mathrm{H})$, $7.8(\mathrm{~d}, 1 \mathrm{H}), 8.4(\mathrm{~d}, 1 \mathrm{H}), 8.81(\mathrm{~d}, 1 \mathrm{H})$. CNMR ( d d $^{6}$-DMSO, $400 \mathrm{mHz}$ ) : 42, 64, 106, 121, 122, 124, 124.5, 126,130, 135, 138,139, 145, $148,164,173$.

ii. 3-(ethylsulfonyl)-5-(2-((5-(1-(4-(trifluoromethyl) phenyl)-1H-imidazol-5-yl)quinolin-8-yl)oxy)ethylidene) thiazolidine-2,4-dione(8b): M.p.:88-290 ${ }^{\circ} \mathrm{C}$. IR (KBr, cm-1): 3040, 3025, 1730, 1645,1603, 1325, 1075,772,715 620,. ${ }^{1}$ HNMR (

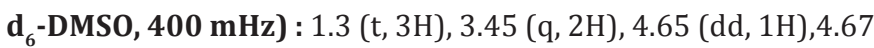
(dd,1H), 6.8 (dd, 1H), $7.13(\mathrm{~m}, 2 \mathrm{H}), 7.32(\mathrm{~d}, 2 \mathrm{H}), 7.65(\mathrm{~m}, 4 \mathrm{H}), 7.8$ (d, 1H), $8.41(\mathrm{~d}, 1 \mathrm{H}), 8.82(\mathrm{~d}, 1 \mathrm{H}) \cdot{ }^{13}$ CNMR ( d $\mathbf{d}^{6}$-DMSO, $400 \mathrm{mHz}$ ) : $10,52,63,106,121,122,124,124.5,126,130,135,138,139$, $145,148,164,173$.

iii. 3 - tosyl- 5 - ( 2 - ( ( 5 - (1 - ( 4 - (trifluoromethyl) phenyl)-1H-imidazol-5-yl)quinolin-8-yl)oxy) ethylidene) thiazolidine-2,4-dione(8c): M.p. 300-302 ${ }^{\circ} \mathrm{C}$. IR (KBr, cm-1): 3050, 3030, 1735, 1640,1603, 1325, 1075,770,715 620. ${ }^{1}$ HNMR ( d d $\left.^{-D M S O}, 400 \mathrm{mHz}\right): 2.3(\mathrm{~s}, 3 \mathrm{H}), 4.68(\mathrm{dd}, 1 \mathrm{H}), 4.69$ (dd, $1 \mathrm{H}), 6.8(\mathrm{dd}, 1 \mathrm{H}), 7.13(\mathrm{~m}, 2 \mathrm{H}), 7.32(\mathrm{~d}, 2 \mathrm{H}), 7.4(\mathrm{~d}, 2 \mathrm{H}), 7.65(\mathrm{~m}$, 4H),7.7 (d, 2H), $7.8(\mathrm{~d}, 1 \mathrm{H}), 8.42(\mathrm{~d}, 1 \mathrm{H}), 8.85(\mathrm{~d}, 1 \mathrm{H}) .{ }^{13} \mathbf{C N M R}$ (d d $^{6}$-DMSO, 400 mHz) : 20, 64, 107, 121, 122, 123, 124, 124.5, 126,128, 130,132, 133 135, 138,139, 145, 148, 155, 165, 175.

iv. 3 -((4-chlorophenyl)sulfonyl) -5 - (2- ( (5- (1- (4(trifluoromethyl)phenyl)-1H-imidazol-5-yl)quinolin-8yl)oxy)ethylidene)thiazolidine-2,4-dione (8d): M.p. 290$292{ }^{\circ} \mathrm{C}$. IR (KBr, cm-1): 3070, 3020, 1720, 1620,1530,1325, 1080,770,720, 655. ${ }^{1}$ HNMR ( d 6 -DMSO, 400 mHz) : 4.68(dd, H), $4.69(\mathrm{dd}, 1 \mathrm{H}), 6.85(\mathrm{dd}, 1 \mathrm{H}), 7.15(\mathrm{~m}, 2 \mathrm{H}), 7.28(\mathrm{~d}, 2 \mathrm{H}), 7.4(\mathrm{~d}$, $2 \mathrm{H}), 7.63(\mathrm{~m}, 6 \mathrm{H}), 7.8(\mathrm{~d}, 2 \mathrm{H}), 7.95(\mathrm{~d}, 1 \mathrm{H}), 8.5(\mathrm{~d}, 1 \mathrm{H}), 8.87(\mathrm{~d}, 1 \mathrm{H})$. ${ }^{13}$ CNMR ( d -DMSO, $400 \mathrm{mHz}$ ) : 64, 108, 121, 122, 123, 124, 124.5, 126,128, 130,132, 133 135, 139, 145, 149, 155, 162, 173.

v. $\quad 3$-((4-bromophenyl) sulfonyl) -5 - (2- ( (5- (1- (4(trifluoromethyl)phenyl)-1H-imidazol-5-yl)quinolin-8yl)oxy)ethylidene)thiazolidine-2,4-dione (8e): M.p. 298$300{ }^{\circ} \mathrm{C}$. IR (KBr, cm-1): 3080, 3025, 1720, 1623,1530,1325, 1085,770,725, 655. ${ }^{1}$ HNMR ( d ${ }^{6}$-DMSO, 400 mHz) : 4.68(dd, $1 \mathrm{H}), 4.69(\mathrm{dd}, 1 \mathrm{H}), 6.87(\mathrm{dd}, 1 \mathrm{H}), 7.18(\mathrm{~m}, 2 \mathrm{H}), 7.28(\mathrm{~d}, 2 \mathrm{H}), 7.6(\mathrm{~m}$, 4H), 7.88(m, 5H), $8.4(\mathrm{~d}, 1 \mathrm{H}), 8.8(\mathrm{~d}, 1 \mathrm{H}) .{ }^{13}$ CNMR (d6-DMSO, 400 mHz): 64.2, 107, 121, 122, 123, 124, 124.5, 126,128, 130,132, 134, 136, 139, 146, 149, 155, 163, 174. 


\section{Organic and Medicinal Chemistry International Journal}

vi. 3 - ( (4-nitrophenyl) sulfonyl) - 5 - ( 2 - ( ( 5 - (1 - ( 4 (trifluoromethyl)phenyl)-1H-imidazol-5-yl)quinolin-8-yl) oxy)ethylidene)thiazolidine-2,4-dione(8f): $\quad$ M.p. 305-308 ${ }^{\circ} \mathrm{C}$. IR (KBr, cm-1): 3070, 3028, 1720, 1623,1535, 1400, 1328,

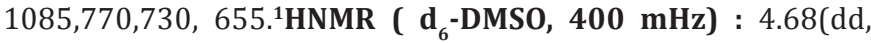
$1 \mathrm{H}), 4.69(\mathrm{dd}, 1 \mathrm{H}), 6.87(\mathrm{dd}, 1 \mathrm{H}), 7.18(\mathrm{~m}, 2 \mathrm{H}), 7.28(\mathrm{~d}, 2 \mathrm{H}), 7.6(\mathrm{~m}$, $4 \mathrm{H}), 7.8(\mathrm{~d}, 1 \mathrm{H}), 8.12(\mathrm{~d}, 2 \mathrm{H}), 8.4(\mathrm{~m}, 3 \mathrm{H}), 8.85(\mathrm{~d}, 1 \mathrm{H}) .{ }^{13} \mathbf{C N M R}$ ( d -DMSO, $400 \mathrm{mHz}$ ) : 64.2, 107, 121, 122, 123, 124, 124.5, $126,128,130,132,134,136,139,142,146,149,151155,164$, 174.5 .

\section{Conclusion}

In this research work we successfully synthesized and characterization, mannich bases of quinoline attached imidazoline thiozolidine 2,4-one derivatives. We are planning to these derivatives check for biological evolution. The biological evolution details will include next journal.

\section{Acknowledgement}

I sincerely thankful to my guide and co-workers, and Department of Chemistry, Sri Krishna devaraya University, (Anantapur,) and Gvk Bio Sciences for providing laboratory and analytical facilities.

\section{References}

1. Ioana Mirela Vasincu, Maria Apotrosoaei, Andreea-Teodora Panzariu (2014) Synthesis and Biological Evaluation of New 1,3-Thiazolidine-4one Derivatives of 2-(4-Isobutylphenyl)propionic Acid. Molecules 19: 15005-15025.

2. Navriti Chadha, Malkeet Singh Bahia, Maninder Kaur, Om Silakari (2015) Bioorganic \& Medicinal Chemistry 23: 2953-2974.

3. Ivanildo Mangueira da Silva, Joao da Silva Filho, Priscila Brandão Gomes da Silva (2014) Synthesis and Antimicrobial Activities of 5-Arylidenethiazolidine-2,4-dione Derivatives. Med Research international 2014: $1-8$.

4. H Sharghi, R Khalifeha, F Moeinia, MH Beyzavia, A Salimi Benic, MM Doroodmand (2011) Mannich Reaction of Secondary Amines, Aldehydes and Alkynes in Water Using $\mathrm{Cu} / \mathrm{C}$ Nanoparticles as a Heterogeneous Catalyst. J Iran Chem Soc 8: 89-103.
5. Archana Kapoor, and Neha Khare (2016) Anti-hyperglycemic evaluation of 2,4-thiazolidinedi one and rhodanine derivatives. Der Pharmacia Lettre 8(12):143-148.

6. Andreea-Teodora Panzariu, Maria Aprotosoaei, Lenuta profire (2016) Chem Cent J. 10.

7. Faiyazalam M Shaikh, Navin B Patel, Dhanji Rajani (2013) Indian jounal of pharmacy and Biotecnology 1: 496.

8. Devprakash, Udaykumar A Bhoi (2011) A complete review of thiazolidine-4-ones. Journal of Pharmacy Research 4 (7): 2436-2440.

9. (1990) European Journal of Medicinal chemistry 25(7): 569-579.

10. BA Bhaviskar, SS Khadabadi, SL Deore (2013) Journal of Chemistry 2013 (2013): 1-6.

11. Tribhuvan Singh, Deepak Khobragade (2014) Synthesis and Evaluation of Thiazolidine-4-One for their Antibacterial Activity. Journal of Pharmaceutical Science and Bioscientific Research 4(1): 110-113.

12. Kishan D Patel, Chhaganbhai N Patel, and Grishma M Patel (2016) Microwave Assisted Synthesis and Antidiabetic Activity of Novel 5-[4-(Substituted) Benzylidine]Thiazolidine-2,4-Dione. Med Chem (Los Angeles) 6-10.

13. Mithun Rudrapal, Dipak Chetia (2016) Asian Journal of Organic and Medicinal Chemistry, 1(2): 53-54

14. Arthur Harutyunyan (2017) Organic and Medicinal Chemistry IJ 3(1): $1-2$

15. Prajwal L Lobo, Boja Poojary, Manjunatha K, Prathibha A, N Suchetha Kumari, et al. (2012) Novel thiazolidine-2,4-dione mannich bases:Synthesis, characterization and antimicrobial activity. Der Pharma Chemica 4 (3): 867-871.

16. Haitao Ji, Qing Jing, Jinwen Huang, Richard B Silverman (2012) Tetrahedron 68(5): 1359-1366.

17. Nicolas Primas, Pierre Verhaeghe, Anita Cohen, Charline Kieffer Aurélien Dumètre, et al. (2012) A New Synthetic Route to Original Sulfonamide Derivatives in 2-Trichloromethylquinazoline Series: A Structure-Activity Relationship Study of Antiplasmodial Activity. Molecules 17: 8105-8117.

18. Faiyazalam, M Shaikh, Navin B Patel, Dhanji Rajani (2013) Indian Journal of Research in Pharmacy and Biotechnology 1(4): 496.

19. Archana Kapoor, Neha Khare (2016) Anti-hyperglycemic evaluation of 2,4-thiazolidinedione and rhodanine derivatives. Der Pharmacia Lettre 8(12): 143-148.

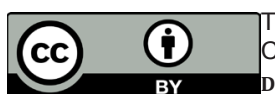

This work is licensed under Creative Commons Attribution 4.0 License DoI: 10.19080/OMCIJ.2017.03.555607

\section{Your next submission with Juniper Publishers will reach you the below assets}

- Quality Editorial service

- Swift Peer Review

- Reprints availability

- E-prints Service

- Manuscript Podcast for convenient understanding

- Global attainment for your research

- Manuscript accessibility in different formats

( Pdf, E-pub, Full Text, Audio)

- Unceasing customer service

Track the below URL for one-step submission

https://juniperpublishers.com/online-submission.php 